OPEN ACCESS

Edited by:

Libero Vitiello,

University of Padua, Italy

Reviewed by:

Paola Braghetta,

University of Padua, Italy

Xu Yan,

Victoria University, Australia

*Correspondence:

Linda Popplewell

linda.popplewel/@rhul.ac.uk

${ }^{\dagger}$ These authors have contributed equally to this work

Specialty section:

This article was submitted to Clinical and Translational Physiology, a section of the journal Frontiers in Physiology

Received: 19 December 2019 Accepted: 17 February 2020 Published: 05 March 2020

Citation: Harish P, Forrest L, Herath S,

Dickson G, Malerba A and Popplewell L (2020) Inhibition of Myostatin Reduces Collagen Deposition in a Mouse Model of Oculopharyngeal Muscular Dystrophy (OPMD) With Established Disease. Front. Physiol. 11:184. doi: 10.3389/fphys.2020.00184

\section{Inhibition of Myostatin Reduces Collagen Deposition in a Mouse Model of Oculopharyngeal Muscular Dystrophy (OPMD) With Established Disease}

\author{
Pradeep Harish, Leysa Forrest, Shanti Herath, George Dickson, Alberto Malerba' and \\ Linda Popplewell*t
}

Department of Biological Sciences, Centre of Gene and Cell Therapy and Biomedical Sciences, Royal Holloway, University of London, Egham, United Kingdom

Background: Oculopharyngeal muscular dystrophy (OPMD) is a late-onset muscle disease presented by ptosis, dysphagia, and limb weakness. Affected muscles display increased fibrosis and atrophy, with characteristic inclusion bodies in the nucleus. Myostatin is a negative regulator of muscle mass, and inhibition of myostatin has been demonstrated to improve symptoms in models of muscular dystrophy.

Methods: We systemically administered a monoclonal antibody to block myostatin in the A17 mouse model of OPMD at 42 weeks of age. The mice were administered a weekly dose of $10 \mathrm{mg} / \mathrm{kg}$ RK35 intraperitonially for 10 weeks, following which serum and histological analyses were performed on muscle samples.

Results: The administration of the antibody resulted in a significant decrease in serum myostatin and collagen deposition in muscles. However, minimal effects on body mass, muscle mass and myofiber diameter, or the density of intranuclear inclusions (INIs) (a hallmark of disease progression of OPMD) were observed.

Conclusion: This study demonstrates that inhibition of myostatin does not revert muscle atrophy in a mouse model with established OPMD disease, but is effective at reducing observed histological markers of fibrosis in the treated muscles.

Keywords: OPMD, myostatin, antibody, RK35, muscular dystrophy

\section{INTRODUCTION}

Oculopharyngeal muscular dystrophy (OPMD) is a late onset muscle wasting disease, whose clinical diagnosis is often compounded by the onset of sarcopenia associated muscle loss (Tome et al., 1997; Blumen et al., 2000; Trollet et al., 2010). The primary clinical indications for the disease involves ptosis, dysphagia, and proximal limb weakness. The genetic basis of the disease revolves around a mutation in the $P A B P N 1$ gene whose product regulates poly (A) tail length on mRNAs, controls the use of alternative polyadenylation (APA) sites, and influences pre-mRNA splicing among other roles (Harish et al., 2015). In OPMD, mutated PABPN1 has a poly-alanine 
expansion at the $\mathrm{N}$ terminus of the protein, resulting in 11-18 repeats instead of the normal 10 present in unaffected individuals (Brais et al., 1998; Blumen et al., 2000). The alanine expansion results in protein misfolding and consequent accumulation in the nuclei as intranuclear inclusion bodies (INI) (Harish et al., 2018). These INI bodies also sequester other molecules such as poly(A)-containing RNA, various transcription factors of the proteasome ubiquitin pathway (ubiquitin and $20 \mathrm{~S}$ catalytic proteasomal subunit), molecular chaperones (HDJ-1, HSP70), heterogeneous nuclear ribonucleoprotein A1 (HNRPA1) and arginine methyltransferares (Harish et al., 2018). The sequestration of these proteins may induce defects in transcriptomic or protein folding pathways (Tavanez et al., 2009; Malerba et al., 2017). Current methods to ameliorate disease symptoms are surgical in nature, however, various small molecule and gene therapy strategies have been proposed that directly or indirectly target the INI bodies (Harish et al., 2018). Concordant with other muscular dystrophies, moderate muscle atrophy (especially in non-somitically derived muscles) has also been described in patients with OPMD (Schmitt and Krause, 1981; Little and Perl, 1982), and hence therapeutic agents that target muscle mass may ameliorate symptoms in this disease state.

Myostatin is a known regulator of muscle mass and has been examined as a therapeutic target to ameliorate symptoms of dystrophy, cachexia, and sarcopenia (Rodgers and Garikipati, 2008; Sartori et al., 2013; Mouisel et al., 2014). While primary myostatin signaling is effected as a balance between the bone morphogenetic protein (BMP) and activing receptor IIB (ACTRIIB) signaling pathways, secondary signaling mechanisms also influence cell growth via interactions with the IGF-1, p21/Cdk, Wnt signaling pathways (Rodgers and Garikipati, 2008; McPherron, 2010; Sartori et al., 2013). Studies in myostatin null mice report an increased bone mineral density (as compared to wild-type controls) and ejection fraction, resistance to diet induced obesity, dyslipidemia, atherogenesis, hepatic steatosis and macrophage infiltration, besides a substantial improvement in muscle mass (White and LeBrasseur, 2014). Inhibition of myostatin on disease progression has been studied in aged $m d x$ mice (modeling Duchenne muscular dystrophy) and C57 (wildtype) model systems utilizing various strategies, and report variable levels of efficacy (LeBrasseur et al., 2009; Murphy et al., 2010; Arounleut et al., 2013). Unsurprisingly, a variety of strategies to disrupt myostatin signaling are in preclinical and clinical development, including but not limited to propeptide, gene therapy, gene editing, ligand traps, and monoclonal antibodies (Wagner et al., 2002, 2008; Bogdanovich et al., 2005; Mendell et al., 2015; Bhattacharya et al., 2017; Campbell et al., 2017).

We recently published that immunological blockade of myostatin in young (12 week old) A17 mice improved muscle mass, muscle force, and reduced collagen deposition (Harish et al., 2019). The A17 model mouse, which expresses a bovine $\exp P A B P N 1$ transgene (17 expanded alanine residues driven by a human alpha actin promoter), has been used routinely in various studies as it models OPMD disease progression on a cellular level (Trollet et al., 2010).
In this current study, we utilize the same treatment regimen in relatively older OPMD mice to assess the impact of myostatin inhibition in models with pre-existing muscle atrophy. Here, we show that administration of the anti-myostatin monoclonal antibody RK35 to 42 week old A17 mice for a period of 10 weeks, while not affecting accumulation of intranuclear aggregates, or loss of body mass, muscle atrophy, or muscle strength, does, however, reduce deposition of fibrotic collagen proteins. This would suggest the proposed anti-myostatin therapy may have a role as an adjuvant treatment option in established disease states in addition to gene therapy or small molecule strategies to ameliorate disease symptoms and presents future avenues to be investigated (Malerba et al., 2017, 2019a).

\section{MATERIALS AND METHODS}

\section{Animal Handling}

A17 and FvB mice were bred in-house and all mice were housed individually with food and water ad libitum in a minimal disease facility at Royal Holloway, University of London (Davies et al., 2005). Individual mice were identified by ear-notching at about 4 weeks of age. Due to the heterozygous nature of the disease model, the OPMD mice were analyzed to confirm the genotype by PCR as described previously (Trollet et al., 2010). 42 week old male mice were weighed prior to each injection, at the same time of day. Initial body weights were used to evenly distribute animals among cohorts to ensure equivalent average body weights prior to the commencement of experimental protocols. In this experiment, we administered the anti-myostatin blocking antibody RK35 (Pfizer, United States) diluted in sterile saline (Sigma-Aldrich, United Kingdom) for a final volume of $200 \mu \mathrm{l}$. The antibody was injected weekly at $10 \mathrm{mg} / \mathrm{kg}$ i.p. for 10 weeks into A17 and FvB mice [9 A17 mice treated with the antimyostatin antibody RK35 (A17 + RK35), 8 A17 mice treated with saline as vehicle control (A17 + saline), $10 \mathrm{FvB}$ mice treated with the antibody RK35 (FvB + RK35), and 10 FvB mice treated with saline as a strain control $(\mathrm{FvB}+$ saline $)]$. In vivo experiments were conducted under statutory Home Office recommendation; regulatory, ethics, and licensing procedures and the Animals (Scientific Procedures) Act 1986 (Project License 36A9994E).

\section{Sample Collection, Processing and Immunological Detection of Serum Myostatin}

Mice were euthanized 1 week after the last injection of RK35 at the 53rd week of age, and the Tibialis Anterior (TA) muscle was harvested, weighed and mounted in O.C.T. compound (Thermo Fisher Scientific, United Kingdom), frozen in 2-methylbutane (isopentane) chilled with liquid nitrogen, and stored at $-80^{\circ} \mathrm{C}$. Blood was extracted by cardiac puncture, allowed to clot overnight at $4^{\circ} \mathrm{C}$, serum extracted and spun down successively with increasing speeds at $1000 \mathrm{~g}, 2000 \mathrm{~g}$, and $3500 \mathrm{~g}$ to remove residual cells. The serum sample thus collected was stored at $-80^{\circ} \mathrm{C}$. Serum samples were then subject to activation (R\&D Systems, United States, DY010) and sandwich ELISA (R\&D 
Systems, United States, DGDF80) to detect serum myostatin as per manufacturer's recommended protocol. Additional samples from mice subject to the same treatment regimen from 12 weeks of age obtained via a previously conducted experiment were also used in this study (Harish et al., 2019).

\section{Histological and Immunohistochemical Analysis}

Transverse sections of the tissue were sectioned at 10-12 different intervals at a thickness of $10 \mu \mathrm{m}$, along the length of the muscle, allowing the maximal cross-sectional area (CSA) to be determined, the sections were mounted on coated slides (VWR International, United Kingdom), and stored at $-80^{\circ} \mathrm{C}$. Sections were then air-dried, fixed, stained with anti-PABPN1 (rabbit monoclonal, diluted 1:100, Abcam ab75855, overnight at $4^{\circ} \mathrm{C}$ ), anti-laminin (rat monoclonal, diluted 1:800, SigmaAldrich L0663, $1 \mathrm{~h}$ at room temperature) and anti- collagen VI (rabbit polyclonal, Abcam ab6588, 1:200, 1 h room temperature) antibodies using previously established protocols (Malerba et al., 2017). Slides were stained with DAPI to visualize nuclei and coverslips were mounted using mounting medium (Vector Labs, United States). Whole muscle images (for laminin based fiber morphometry) or random fields (for PABPN1 and collagen immunostaining) were captured using a microscope (Zeiss, United Kingdom). For analysis of fiber morphometry, the median minimal feret's diameter from 1000 randomly selected fibers were determined for TA muscles for each individual animal analyzed (Harish et al., 2019; Malerba et al., 2019a). For picrosirius red staining, sections were air dried, fixed in PFA 4\%, and stained in a $0.3 \%$ solution of Sirius red in saturated aqueous picric acid, followed by a treatment with $0.5 \%$ acetic acid. Imaging of sirius red staining was performed under white light to analyze collagen deposition.

\section{Statistical Analyses}

After checking for the conditions of normality and homoscedasticity, a one way ANOVA was performed to compare multiple groups. Multiple comparison were performed with Bonferroni correction to correct for multiple testing used. All statistical techniques were performed using GraphPad Prism v7.00 (GraphPad Software, California, United States).

\section{RESULTS}

\section{Treatment With Anti-myostatin Antibody Has Minimal Effect on Body Mass in 52-Week Old OPMD Mice}

To monitor the effects of inhibiting myostatin in a mouse model of OPMD, where the disease is already established, 8-10 male A17 and FvB mice at 42 weeks of age were weighed and the antimyostatin antibody RK35 (10 mg/kg IP) or an equivalent volume of saline was administered weekly for 10 weeks (Figure 1A).

At the beginning of the study a $10 \%(p<0.05)$ difference in the initial body mass existed between A17 mice and FvB mice (Figure 1B). This difference was maintained throughout the study. The treatment of A17 mice with the antibody caused a non-significant increase in final body mass of $5 \%(p>0.05)$ when compared to the saline treated A17 mice (Figure 1C). The treatment of FvB control mice resulted in no significant changes in body mass observed.

\section{Treatment With Anti-myostatin Antibody Has Minimal Effect on Muscle Mass and Muscle Fiber Diameter, and Intranuclear Inclusion Density in 52-Week Old OPMD Mice}

When examining the change in muscle mass, we detected a significant increase of $20 \%(p<0.01)$ in raw muscle masses of A17 and FvB muscles treated with saline (Figure 2A). No difference in the mass of the TA (normalized to the initial body mass) was found between the untreated A17 and FvB mice (Figure 2B). It should be noted that the administration of the antibody RK35 resulted in no changes to either the normalized
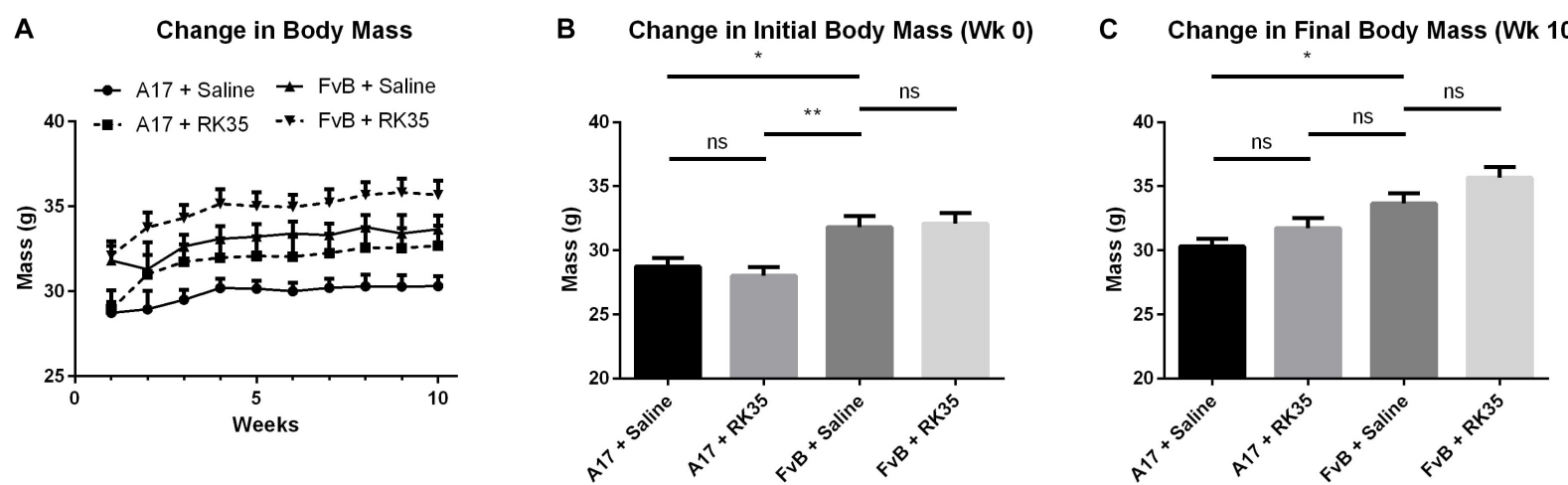

FIGURE 1 | Treatment with RK35 antibody does not affect body mass in 52 week old A17 mice: Mice $(n=8-10)$ were weighed and administered a weekly regimen of either saline or the RK35 antibody (10 mg/kg i.p.) for 10 weeks from the 42 nd week of age. (A) The average body mass per group plotted against weeks shows that body masses $\mathbf{( B )}$ are significantly different at the beginning of the experiment and $\mathbf{( C )}$ the difference is maintained after 10 weeks of treatment. Data presented as mean with SEM, with $p$-values obtained by ANOVA after a Bonferroni correction $\left({ }^{*} p<0.05 ;{ }^{* *} p<0.01\right)$. 

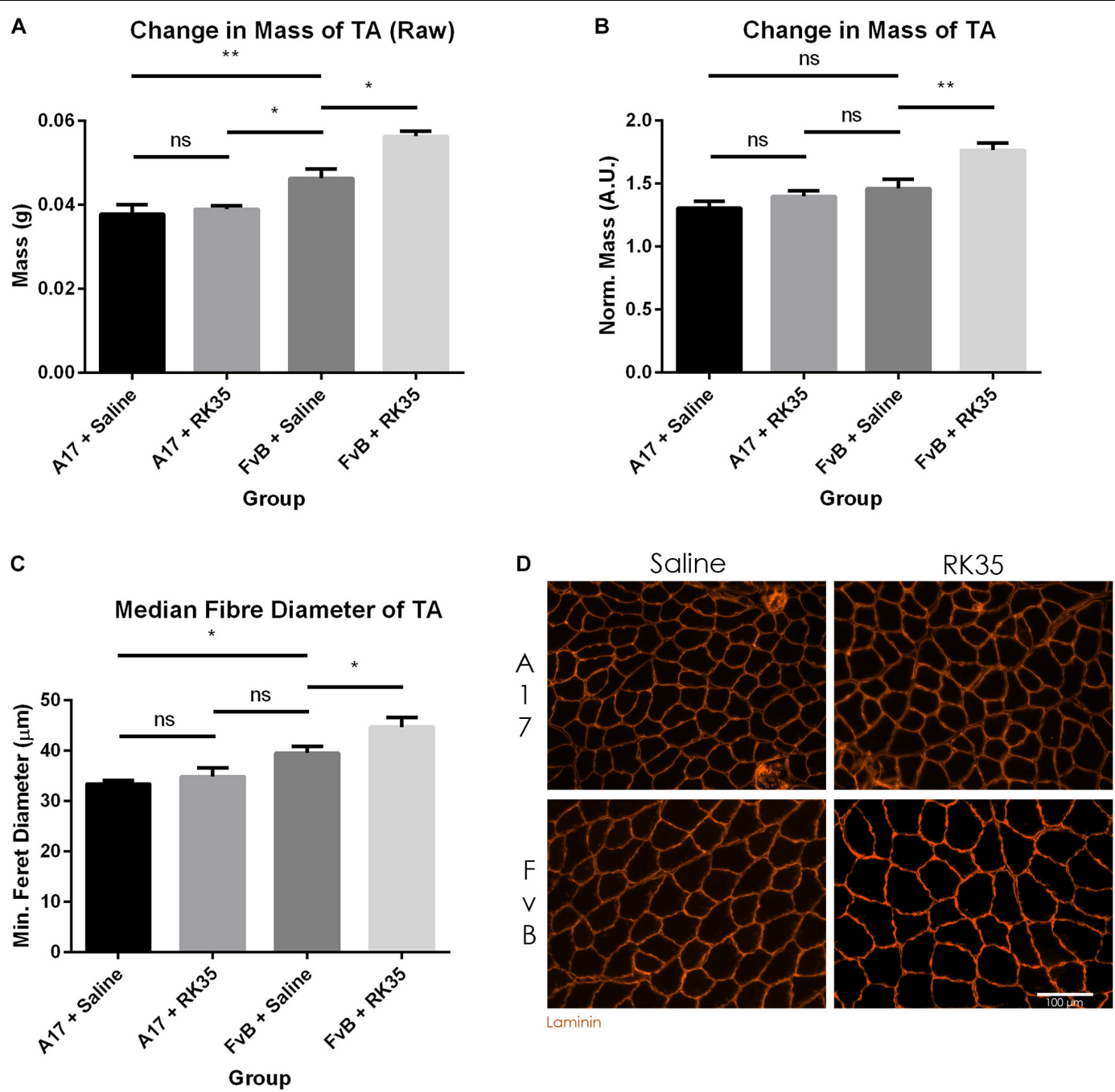

FIGURE 2 | Treatment with RK35 antibody has does not affect muscle mass and myofiber diameter in OPMD mice: Mice were administered with a weekly regimen of either saline or the RK35 antibody (10 mg/kg i.p.) for 10 weeks from 42-weeks of age. The TA was sampled and (A) raw muscle mass or (B) muscle mass normalized to the initial body mass is presented. Next, muscle samples from five randomly selected mice per group were sectioned and immunostained for laminin. (C) 1000 fibers were randomly selected for the TA, and the median minimum feret diameter was analyzed. (D) Representative images used to generate myofiber diameter data are shown on the bottom right panel with the scale bar representing $100 \mu \mathrm{m}$. The FvB mice treated with the antibody consistently displayed an increased muscle mass and myofiber diameter, with no changes observed in the A17 mice. The average of median minimum feret diameter per group was plotted, Data presented as mean with SEM, with $p$-values obtained by ANOVA after a Bonferroni correction $\left({ }^{*} p<0.05 ;{ }^{* *} p<0.01\right)$.

or raw muscle mass of the TA in the A17 mice when compared to saline controls. However, a significant increase in muscle mass in the treated FvB controls [19\% $(p<0.05)$ increase in normalized and raw muscle masses] was observed when compared to the untreated FvB mice, demonstrating the functionality of the administered antibody and suggesting that this treatment was not able to reverse established muscle atrophy (Figures 2A,B).

To better characterize the muscle atrophy in aged A17 mice, we analyzed the myofiber size. The decrease in muscle mass in the A17 mice was accompanied by a reduction of myofiber diameter in the TA by $18 \%(p<0.05)$ when compared to FvB mice (Figures 2C,D). The administration of RK35 to A17 mice resulted in a minimal increase in myofiber diameter of the TA (Figure 2C). In accordance with the increase in muscle mass observed in FvB mice, the myofiber diameter in TA muscles of age-matched FvB controls increased by $13 \%(p<0.05)$ compared to those of saline-treated FvB mice (Figure 2C). Finally, the administration of the RK35 antibody did not affect the levels of INIs in the TA in this study (Supplementary Figure S1).

\section{Anti-myostatin Antibody Significantly Reduces Collagen Deposition in A17 Mice}

We next examined the effect of inhibition of myostatin on the deposition of collagen proteins as histopathological markers of fibrotic tissue deposition in the muscles. Untreated A17 mice displayed a significantly increased collagen deposition when 
A

Change in Collagen VI deposition

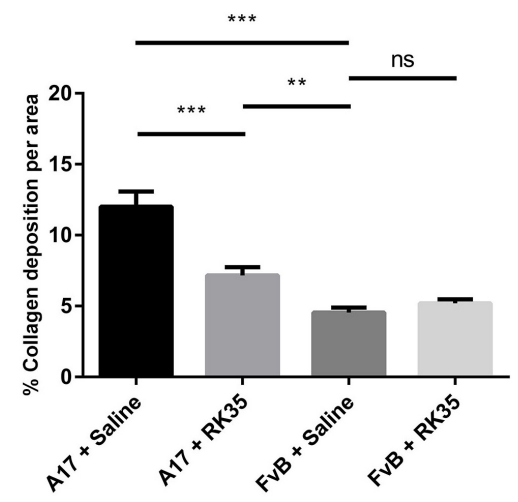

C
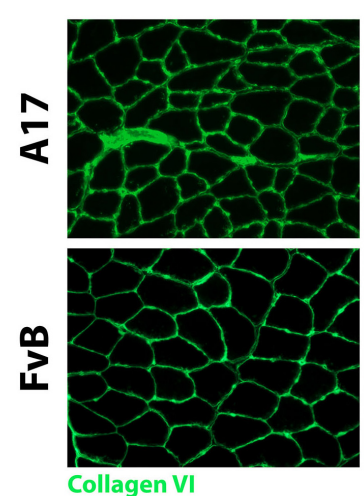

RK35
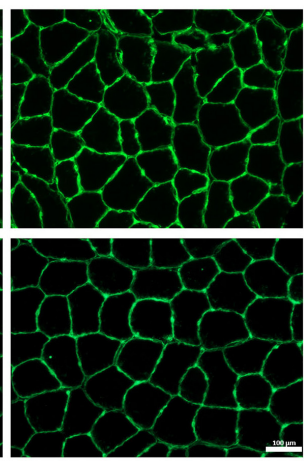

B Change in Collagen deposition

(visualised by sirius red staining)

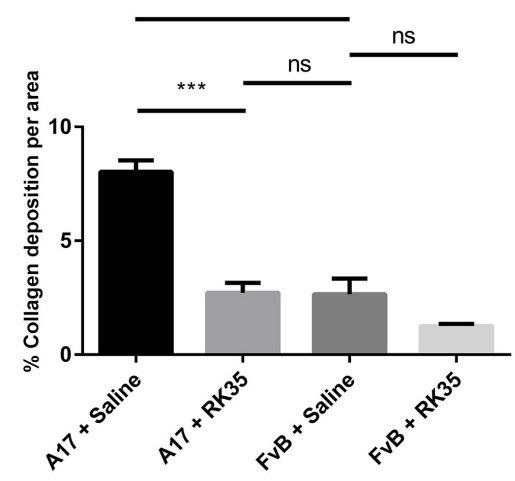

D Saline
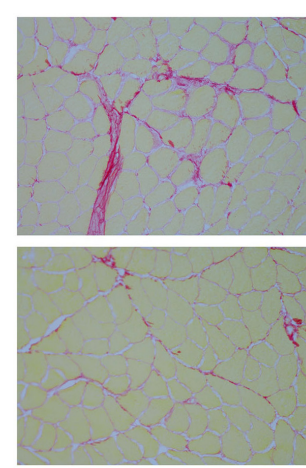

Sirius Red

FIGURE 3 | Treatment with RK35 antibody reduces collagen deposition in OPMD mice: Mice were administered with a weekly regimen of either saline or the RK35 antibody (10 mg/kg i.p.) for 10 weeks from 42-weeks of age. Five randomly selected whole TA muscle sections from all groups were stained for (A) collagen VI and (B) picrosirius red, and five random fields were imaged and analyzed for the percentage area of collagen staining. (C,D) Representative images used to generate data are shown below the respective graphs, with the scale bar representing $100 \mu \mathrm{m}$. The mice treated with the antibody consistently displayed a reduced collagen deposition, with the area of collagen in the disease model normalized to wild-type levels. The average area per group is plotted, bars representing SEM, with $p$-values obtained by ANOVA after a Bonferroni correction $\left({ }^{* \star} p<0.01 ;{ }^{* \star *} p<0.001\right)$.

compared to control $\mathrm{FvB}$ mice [with the control mice displaying $60 \%(p<0.05)$ less collagen deposition than the A17 mice] (Figures 3A,C). Inhibition of myostatin by administered RK35 antibody reduced deposition of collagen VI in the TA muscle of A17 mice by $40 \%(p<0.001)$ and general collagen deposition as visualized by Sirius red staining by $29 \%(p<0.01)$ when compared to untreated A17 mice (Figures 3B,D). This reduction in collagen deposition (as determined by Sirius red staining) in treated A17 mice was particularly marked since they were ameliorated to the levels seen in the FvB controls (4\% difference, $p>0.05$ ) (Figure 3B).

\section{Treatment With Anti-myostatin Antibody Significantly Reduces Serum Myostatin 52-Week Old OPMD Mice}

We next performed a sandwich ELISA in order to quantify the efficacy of the anti-myostatin antibody RK35 used in this study to reduce the levels of serum myostatin. To establish any age-dependency of response, we utilized serum samples from 52 week old or 12 week old A17 and FvB mice treated systemically with either saline or RK35 for 10 weeks, at $10 \mathrm{mg} / \mathrm{kg}$ i.p. $(n=5$ per group) obtained from a previous study (Harish et al., 2019).

When considering the control samples from the 22 week old mice, the levels of serum myostatin are $40 \%$ higher $(p<0.01)$ in the FvB mice than the A17 mice (Figure 4A). Effective reduction of myostatin using the RK35 antibody levels below the detectable limits of the ELISA is evident for both A17 and FvB young mice (Figure 4B). For the older mice, while no significant difference was observed in serum myostatin levels between 52 week old A17 and $\mathrm{FvB}$ mice, the treatment with the anti-myostatin antibody RK35 again resulted in reduction of serum myostatin to below detectable levels for both strains of mice (Figure 4A). When comparing the levels of serum myostatin between the two age groups, no significant difference was observed between untreated A17 mice at 22 weeks of age or 52 weeks of age. Similarly, no 


\section{A after administration of RK35}

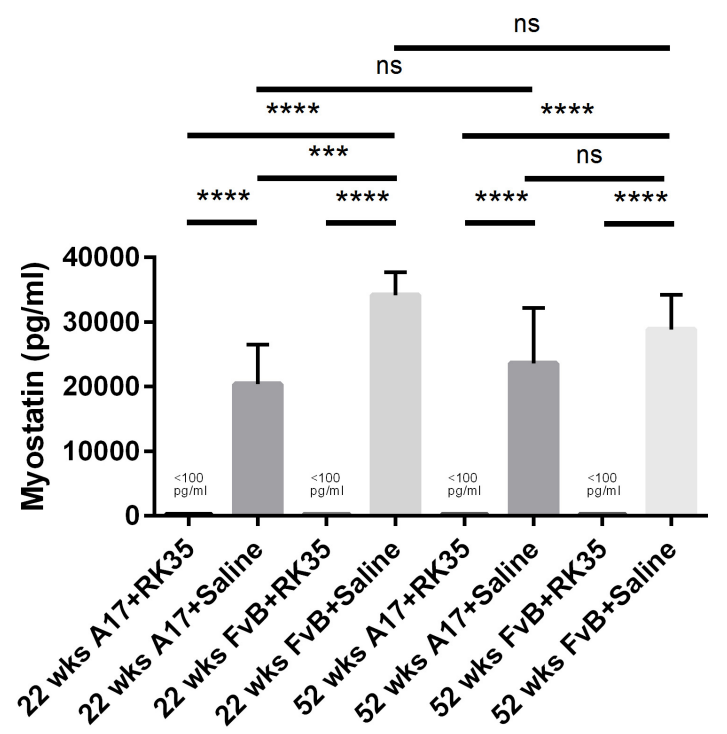

B Optical densities of serum
myostatin levels after RK35 treatment

ns

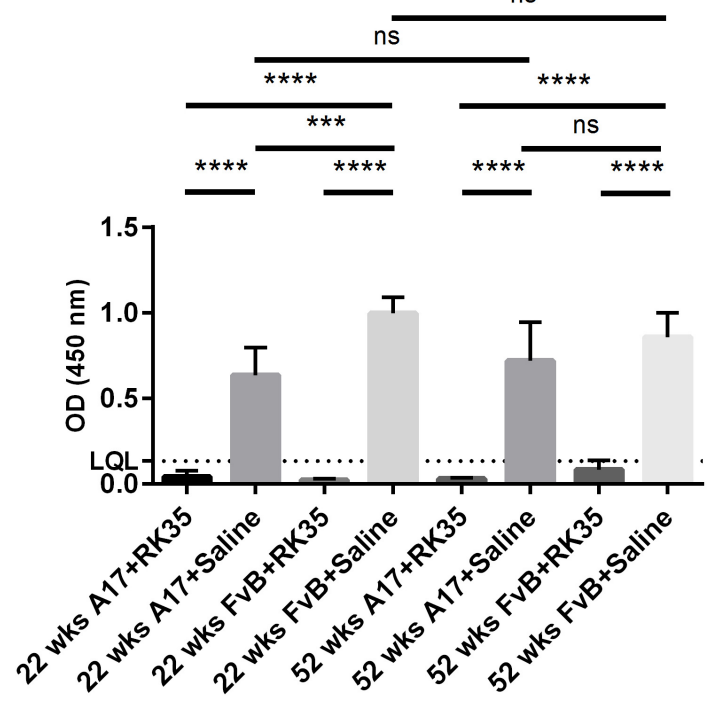

FIGURE 4 | Treatment with RK35 significantly reduces levels of serum myostatin: Serum samples from mice $(n=5)$ were subject to a weekly regimen of either saline or the anti-myostatin RK35 antibody i.p. for 10 weeks from 42 weeks of age, or samples were utilized from a previously published study (Harish et al., 2019) were subject to a sandwich ELISA to detect myostatin. (A) The administration of the antibody resulted in the reduction of serum myostatin below the lower quantitation limit (LQL) of $100 \mathrm{pg} / \mathrm{ml}$. (B) Optical densities corrected for background readings are plotted for each group analyzed. Data presented as mean with SEM, with $p$-values obtained by ANOVA after a Bonferroni correction $\left({ }^{\star \star \star} p<0.001,{ }^{\star \star \star \star} p<0.0001\right)$.

significant difference was observed between untreated FvB mice at 22 weeks of age or 52 weeks of age (Figure $4 \mathrm{~A}$ ).

\section{DISCUSSION}

Myostatin is a negative regulator of muscle mass (Rodgers and Garikipati, 2008). Subsequently, the most striking result of inhibition of myostatin involves significant increases in muscle mass and muscle fiber diameter (McPherron et al., 1997). Hence, agents that either directly or indirectly disrupt myostatin signaling are being investigated by various pharmaceutical industries as a generic broad-spectrum therapeutic strategy to alleviate muscle loss arising from cachexia, sarcopenia, or muscular dystrophy (Rodgers and Garikipati, 2008; LuNguyen et al., 2015, 2017; Camporez et al., 2016; Andre et al., 2017; Tinklenberg et al., 2017). OPMD is a rare poly-alanine expansion induced protein aggregopathy, and has been modeled in the A17 mouse strain (Davies et al., 2005). It is reported that the disease progression manifests as visible changes in muscle mass at 18 weeks of age in the model mice (Trollet et al., 2010). Recent efforts to ameliorate symptoms have focused on small molecule approaches to target protein aggregation and gene therapy approaches to correct the mutation (Malerba et al., 2017, 2019a,b). In our previous study we showed that the administration of the RK35 antibody counteracted the muscle atrophy and improved histopathological features of the disease (Harish et al., 2019). Screening of therapeutic reagents in relatively younger murine models of late-onset diseases, such as OPMD, presents challenges in translating into clinical trials (Harish et al., 2015). Furthermore, pathogenesis of disease in OPMD mice is markedly different from disease progression in human patients. While the disease profile in the A17 mouse model is present in all skeletal muscles, the etiological profile in humans is localized to the extraocular and pharyngeal muscles, with proximal limb muscle involvement depending on disease progression and severity (Davies et al., 2005; Trollet et al., 2010). Therefore, the rationale behind this study was to assess the efficacy of myostatin inhibition in an OPMD disease model with an established disease state.

The A17 mouse model at 52-weeks of age possesses a lower body and muscle mass than the FvB controls, along with a significantly reduced myofiber diameter and increased collagen deposition. The inhibition of myostatin produced no increase in muscle mass in the A17 model mice, which is in contrast to what we observed in younger A17 mice (Harish et al., 2019). Interestingly, the functionality of the antibody was evident in the healthy 52-week old $\mathrm{FvB}$ control mice, with significantly increased muscle mass and myofiber diameters observed in this study. We originally hypothesized that different levels of serum myostatin between A17 and FvB mice could be implicated in the differing efficacy of myostatin inhibition observed between the two age groups. Indeed, Mariot et al. (2017) have reported that levels of serum myostatin might be directly dependant on type of disease and state of disease progression. We report here that the levels of serum myostatin are significantly lower in 22-week old A17 mice when compared to the FvB controls and this correlates 
well with the results obtained in younger mice (Harish et al., 2019). However, no such difference exists in the 52-weeks cohort.

The mechanistic reason behind this differing effect will need to be investigated further in a future study. We speculate a combined sarcopenic and dystrophic phenotype may irreversibly alter muscle structure and attenuate the efficacy of the therapeutic regimen as compared to models in earlier stages of the disease, thereby preventing regrowth of the muscle mass with myostatin inhibition. For example, we speculate crucial molecules involved in the myostatin pathway (e.g., the activin receptor IIb) or molecular pathways affecting the muscle growth (e.g., Insulin like growth factors) may be dysregulated as result of the OPMD pathology significantly reducing the impact of myostatin inhibition. Alternatively, other molecules which negatively regulate muscle mass (such as activin A), might reduce the efficacy of myostatin knockdown in this older cohort (Latres et al., 2017).

Promisingly, while the untreated A17 mice displayed a marked increased collagen deposition when compared to the untreated FvB controls in the TA, the administration of the treatment regimen reduced the collagen deposition. Pathophysiological fibrosis associated with aging/dystrophy results in sarcolemmal fragility, disturbances in calcium ion homeostasis, inflammation, reduction of motile and contractile functions, and reduction of available tissue for therapeutic intervention (Desguerre et al., 2009; Mann et al., 2011). Attenuation of muscle fibrotic processes may have long term benefits to a dystrophic muscle in terms of promoting regeneration, muscle force transfer, and maintaining structural integrity. Myostatin is a known regulator of fibroblast activation and apoptosis ( $\mathrm{Li}$ et al., 2008), with anti-myostatin agents shown to have an anti-fibrotic effect in other models of muscular dystrophy (Wagner et al., 2002; Li et al., 2012).

It is interesting to note in our studies that while inhibiting myostatin in younger mice improved muscle atrophy and reduced collagen deposition (Harish et al., 2019), the inhibition of myostatin in older mice had only a significant effect on reducing collagen deposition. This underlines the importance of elucidating the mechanism of myostatin inhibition on pre-existing muscle atrophy, as this may have an impact on translating anti-myostatin treatment regimens to the clinic. Indeed, most direct immunological blockers of myostatin activity have had limited success in clinical trials (Wagner et al., 2008; Campbell et al., 2017), with primary endpoints being a readout of lean mass, muscle volume, inflammatory responses or muscle regeneration. It may be informative to verify the therapeutic benefit of

\section{REFERENCES}

Andre, M. S., Johnson, M., Bansal, P. N., Wellen, J., Robertson, A., Opsahl, A., et al. (2017). A mouse anti-myostatin antibody increases muscle mass and improves muscle strength and contractility in the mdx mouse model of Duchenne muscular dystrophy and its humanized equivalent, domagrozumab (PF-06252616), increases muscle volume in cynomolgus monkeys. Skelet. Muscle 7:25. doi: 10.1186/s13395-017-0141-y

Arounleut, P., Bialek, P., Liang, L. F., Upadhyay, S., Fulzele, S., Johnson, M., et al. (2013). A myostatin inhibitor (propeptide-Fc) increases muscle mass and muscle fiber size in aged mice but does not increase bone density or bone strength. Exp. Gerontol. 48, 898-904. doi: 10.1016/j.exger.2013.06.004 the anti-fibrotic action of myostatin inhibition in late-onset muscle diseases.

\section{DATA AVAILABILITY STATEMENT}

All datasets generated for this study are included in the article/Supplementary Material.

\section{ETHICS STATEMENT}

The animal study was reviewed, approved, and performed under regulations and recommendations laid down by the UK Home Office (ASPA 1986).

\section{AUTHOR CONTRIBUTIONS}

$\mathrm{PH}, \mathrm{AM}, \mathrm{LP}$, and GD contributed to the study design. PH, AM, $\mathrm{SH}$, and LF participated in the data collection and interpretation. $\mathrm{PH}$ and AM prepared the manuscript. All authors provided the manuscript review and revisions.

\section{ACKNOWLEDGMENTS}

The authors wish to thank Jane Owens, Michael St-Andre, Carl Morris, and other colleagues at Pfizer for providing the antibody RK35, and for support designing the study.

\section{SUPPLEMENTARY MATERIAL}

The Supplementary Material for this article can be found online at: https://www.frontiersin.org/articles/10.3389/fphys. 2020.00184/full\#supplementary-material

FIGURE S1 | Treatment with RK35 does not affect the amount of intranuclear aggregates: Mice were subject to a weekly regimen of either saline or the anti-myostatin RK35 antibody i.p. for 10 weeks from 42 weeks of age. (A) Five muscle samples from all groups were stained for endogenous PABPN1 after a 1M $\mathrm{KCl}$ treatment with the scale bar representing $50 \mu \mathrm{m}$, and five random fields were imaged and analyzed for the percentage number of positive PABPN1 stains to all myonuclei. (B) The administration of the treatment regimen did not change the number of intranuclear aggregates observed. The average density is plotted, bars representing SEM, with $p$-values obtained by ANOVA after a Bonferroni correction $\left.{ }^{\star \star \star \star} P<0.001\right)$.
Bhattacharya, I., Pawlak, S., Marraffino, S., Christensen, J., Sherlock, S. P., Alvey, C., et al. (2017). Safety, tolerability, pharmacokinetics, and pharmacodynamics of domagrozumab (PF-06252616), an antimyostatin monoclonal antibody, in healthy subjects. Clin. Pharmacol. Drug Dev. 7, 484-497. doi: 10.1002/ cpdd. 386

Blumen, S. C., Korczyn, A. D., Lavoie, H., Medynski, S., Chapman, J., Asherov, A., et al. (2000). Oculopharyngeal MD among bukhara jews is due to a founder (GCG)9 mutation in the PABP2 gene. Neurology 55, 1267-1270. doi: 10.1212/ wnl.55.9.1267

Bogdanovich, S., Perkins, K. J., Krag, T. O. B., Whittemore, A. L., and Khurana, T. S. (2005). Myostatin propeptide-mediated amelioration of dystrophic pathophysiology. FASEB J. 19, 543-549. doi: 10.1096/fj.04-2796com 
Brais, B., Bouchard, J. P., Xie, Y. G., Rochefort, D. L., and Chrtien, N. (1998). Short GCG expansions in the PABP2 gene cause oculopharyngeal muscular dystrophy. Nat. Genet. 18, 164-167. doi: 10.1038/ng0298-164

Campbell, C., McMillan, H. J., Mah, J. K., Tarnopolsky, M., Selby, K., McClure, T., et al. (2017). Myostatin inhibitor ACE-031 treatment of ambulatory boys with Duchenne muscular dystrophy: Results of a randomized, placebocontrolled clinical trial. Muscle Nerve 55, 458-464. doi: 10.1002/mus. 25268

Camporez, J. P., Petersen, M. C., Abudukadier, A., Moreira, G. V., Jurczak, M. J., Friedman, G., et al. (2016). Anti-myostatin antibody increases muscle mass and strength and improves insulin sensitivity in old mice. Proc. Natl. Acad. Sci. U.S.A. 113, 2212-2217. doi: 10.1073/pnas. 1525795113

Davies, J. E., Wang, L., Garcia-Oroz, L., Cook, L. J., Vacher, C., O’Donovan, D. G., et al. (2005). Doxycycline attenuates and delays toxicity of the oculopharyngeal muscular dystrophy mutation in transgenic mice. Nat. Med. 11, 672-677. doi: $10.1038 / \mathrm{nm} 1242$

Desguerre, I., Mayer, M., Leturcq, F., Barbet, P. J., Gherardi, R. K., and Christov, C. (2009). Endomysial fibrosis in duchenne muscular dystrophy: a marker of poor outcome associated with macrophage alternative activation. J. Neuropathol. Exp. Neurol. 68, 762-773. doi: 10.1097/NEN.0b013e3181aa31c2

Harish, P., Dickson, G., and Malerba, A. (2018). Advances in emerging therapeutics for oculopharyngeal muscular dystrophy. Exp. Opin. Orphan Drugs 6, 693-701. doi: 10.1080/21678707.2018.1536542

Harish, P., Malerba, A., Dickson, G., and Bachtarzi, H. (2015). Progress on gene therapy, cell therapy, and pharmacological strategies toward the treatment of oculopharyngeal muscular dystrophy. Hum. Gene Ther. 26, 286-292. doi: 10. 1089/hum.2015.014

Harish, P., Malerba, A., Lu-Nguyen, N., Forrest, L., Cappellari, O., Roth, F., et al. (2019). Inhibition of myostatin improves muscle atrophy in oculopharyngeal muscular dystrophy (OPMD). J. Cachexia Sarcopenia Muscle 10, 1016-1026. doi: $10.1002 /$ jcsm. 12438

Latres, E., Mastaitis, J., Fury, W., Miloscio, L., Trejos, J., Pangilinan, J., et al. (2017). Activin a more prominently regulates muscle mass in primates than does GDF8. Nat. Commun. 8:15153. doi: 10.1038/ncomms15153

LeBrasseur, N. K., Schelhorn, T. M., Bernardo, B. L., Cosgrove, P. G., Loria, P. M., and Brown, T. A. (2009). Myostatin inhibition enhances the effects of exercise on performance and metabolic outcomes in aged mice. J. Gerontol. Ser. A 64, 940-948. doi: 10.1093/gerona/glp068

Li, Z. B., Kollias, H. D., and Wagner, K. R. (2008). Myostatin directly regulates skeletal muscle fibrosis. J. Biol. Chem. 283, 19371-19378. doi: 10.1074/jbc. M802585200

Li, Z. B., Zhang, J., and Wagner, K. R. (2012). Inhibition of myostatin reverses muscle fibrosis through apoptosis. J. Cell Sci. 125, 3957-3965. doi: 10.1242/jcs. 090365

Little, B. W., and Perl, D. P. (1982). Oculopharyngeal muscular dystrophy: an autopsied case from the french-canadian kindred. J. Neurol. Sci. 53, $145-158$.

Lu-Nguyen, N., Malerba, A., Popplewell, L., Schnell, F., Hanson, G., and Dickson, G. (2017). Systemic antisense therapeutics for dystrophin and myostatin exon splice modulation improve muscle pathology of adult mdx mice. Mol. Ther. Nucleic Acids 6, 15-28. doi: 10.1016/j.omtn.2016.11.009

Lu-Nguyen, N. B., Jarmin, S. A., Saleh, A. F., Popplewell, L., Gait, M. J., and Dickson, G. (2015). Combination antisense treatment for destructive exon skipping of myostatin and open reading frame rescue of dystrophin in neonatal mdx mice. Mol. Ther. 23, 1341-1348. doi: 10.1038/mt.2015.88

Malerba, A., Klein, P., Bachtarzi, H., Jarmin, S. A., Cordova, G., Ferry, A., et al. (2017). PABPN1 gene therapy for oculopharyngeal muscular dystrophy. Nat. Commun. 8:14848. doi: 10.1038/ncomms14848

Malerba, A., Roth, F., Harish, P., Dhiab, J., Lu-Nguyen, N., Cappellari, O., et al. (2019a). Pharmacological modulation of the ER stress response ameliorates oculopharyngeal muscular dystrophy. Hum. Mol. Genet. 28, 1694-1708. doi: $10.1093 / \mathrm{hmg} / \mathrm{ddz} 007$

Malerba, A., Roth, F., Strings, V., Harish, P., Suhy, D., Trollet, C., et al. (2019b). Gene therapy for oculopharyngeal muscular dystrophy. Muscle Gene Ther. 2019, 549-564. doi: 10.1007/978-3-030-03095-7_31

Mann, C. J., Perdiguero, E., Kharraz, Y., Aguilar, S., Pessina, P., Serrano, A. L., et al. (2011). Aberrant repair and fibrosis development in skeletal muscle. Skelet. Muscle 1:21. doi: 10.1186/2044-5040-1-21
Mariot, V., Joubert, R., and Hourd, C. (2017). Downregulation of myostatin pathway in neuromuscular diseases may explain challenges of anti-myostatin therapeutic approaches. Nat. Commun. 8:1859. doi: 10.1038/s41467-01701486-4

McPherron, A. C. (2010). Metabolic functions of myostatin and GDF11. Immunol. Endocr. Metab. Agents Med. Chem. 10, 217-231. doi: 10.2174/ 187152210793663810

McPherron, A. C., Lawler, A. M., and Lee, S. J. (1997). Regulation of skeletal muscle mass in mice by a new TGF-beta superfamily member. Nature $387,83-90$. doi: 10.1038/387083a0

Mendell, J. R., Sahenk, Z., Malik, V., Gomez, A. M., Flanigan, K. M., Lowes, L. P., et al. (2015). A phase $1 / 2$ a follistatin gene therapy trial for becker muscular dystrophy. Mol. Ther. 23, 192-201. doi: 10.1038/mt.2014.200

Mouisel, E., Relizani, K., Mille-Hamard, L., Denis, R., and Hourd, C. (2014). Myostatin is a key mediator between energy metabolism and endurance capacity of skeletal muscle. Am. J. Physiol. Regul. Integr. Comp. Physiol. 307, R444-R454. doi: 10.1152/ajpregu.00377.2013

Murphy, K. T., Koopman, R., Naim, T., Lger, B., Trieu, J., Ibebunjo, C., et al. (2010). Antibody-directed myostatin inhibition in 21-mo-old mice reveals novel roles for myostatin signaling in skeletal muscle structure and function. FASEB J. 24, 4433-4442. doi: 10.1096/fj.10-159608

Rodgers, B. D., and Garikipati, D. K. (2008). Clinical, agricultural, and evolutionary biology of myostatin: a comparative review. Endocr. Rev. 29, 513-534. doi: 10.1210/er.2008-0003

Sartori, R., Schirwis, E., Blaauw, B., Bortolanza, S., Zhao, J., Enzo, E., et al. (2013). BMP signaling controls muscle mass. Nat. Genet. 45, 1309-1318. doi: 10.1038/ ng. 2772

Schmitt, H. P., and Krause, K. H. (1981). An autopsy study of a familial oculopharyngeal muscular dystrophy (OPMD) with distal spread and neurogenic involvement. Muscle Nerve 4, 296-305. doi: 10.1002/mus. 880040406

Tavanez, J. P., Bengoechea, R., Berciano, M. T., Lafarga, M., Carmo-Fonseca, M., and Enguita, F. J. (2009). Hsp70 chaperones and type I PRMTs are sequestered at intranuclear inclusions caused by polyalanine expansions in PABPN1. PLoS One 4:e6418. doi: 10.1371/journal.pone.0006418

Tinklenberg, J. A., Siebers, E. M., Beatka, M. J., Meng, H., Yang, L., Zhang, Z., et al. (2017). Myostatin inhibition using mRK35 produces skeletal muscle growth and tubular aggregate formation in wild type and TgACTA1D286G nemaline myopathy mice. Hum. Mol. Genet. 27, 638-648. doi: 10.1093/hmg/ddx431

Tome, F. M., Chateau, D., Helbling-Leclerc, A., and Fardeau, M. (1997). Morphological changes in muscle fibers in oculopharyngeal muscular dystrophy. Neuromuscul. Disord. 7(Suppl. 1), S63-S69.

Trollet, C., Anvar, S. Y., Venema, A., Hargreaves, I. P., Foster, K., Vignaud, A., et al. (2010). Molecular and phenotypic characterization of a mouse model of oculopharyngeal muscular dystrophy reveals severe muscular atrophy restricted to fast glycolytic fibres. Hum. Mol. Genet. 19, 2191-2207. doi: 10.1093/hmg/ ddq098

Wagner, K. R., Fleckenstein, J. L., Amato, A. A., Barohn, R. J., Bushby, K., Escolar, D. M., et al. (2008). A phase I/IItrial of MYO-029 in adult subjects with muscular dystrophy. Ann. Neurol. 63, 561-571. doi: 10.1002/ana.21338

Wagner, K. R., McPherron, A. C., Winik, N., and Lee, S. J. (2002). Loss of myostatin attenuates severity of muscular dystrophy in mdx mice. Ann. Neurol. 52, 832-836. doi: 10.1002/ana.10385

White, T. A., and LeBrasseur, N. K. (2014). Myostatin and sarcopenia: opportunities and challenges-a mini-review. Gerontology 60, 289-293. doi: 10. $1159 / 000356740$

Conflict of Interest: The authors declare that the research was conducted in the absence of any commercial or financial relationships that could be construed as a potential conflict of interest.

Copyright $\odot 2020$ Harish, Forrest, Herath, Dickson, Malerba and Popplewell. This is an open-access article distributed under the terms of the Creative Commons Attribution License (CC BY). The use, distribution or reproduction in other forums is permitted, provided the original author(s) and the copyright owner(s) are credited and that the original publication in this journal is cited, in accordance with accepted academic practice. No use, distribution or reproduction is permitted which does not comply with these terms. 